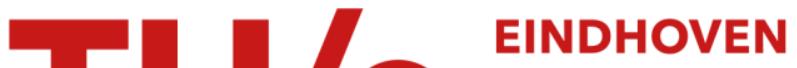 \\ UNIVERSITY OF \\ TECHNOLOGY
}

\section{On modeling of curved jets of viscous fluid hitting a moving surface}

\section{Citation for published version (APA):}

Hlod, A. (2010). On modeling of curved jets of viscous fluid hitting a moving surface. (CASA-report; Vol. 1066). Technische Universiteit Eindhoven.

\section{Document status and date:}

Published: 01/01/2010

\section{Document Version:}

Publisher's PDF, also known as Version of Record (includes final page, issue and volume numbers)

\section{Please check the document version of this publication:}

- A submitted manuscript is the version of the article upon submission and before peer-review. There can be important differences between the submitted version and the official published version of record. People interested in the research are advised to contact the author for the final version of the publication, or visit the $\mathrm{DOI}$ to the publisher's website.

- The final author version and the galley proof are versions of the publication after peer review.

- The final published version features the final layout of the paper including the volume, issue and page numbers.

Link to publication

\section{General rights}

Copyright and moral rights for the publications made accessible in the public portal are retained by the authors and/or other copyright owners and it is a condition of accessing publications that users recognise and abide by the legal requirements associated with these rights.

- Users may download and print one copy of any publication from the public portal for the purpose of private study or research.

- You may not further distribute the material or use it for any profit-making activity or commercial gain

- You may freely distribute the URL identifying the publication in the public portal.

If the publication is distributed under the terms of Article 25fa of the Dutch Copyright Act, indicated by the "Taverne" license above, please follow below link for the End User Agreement:

www.tue.nl/taverne

Take down policy

If you believe that this document breaches copyright please contact us at:

openaccess@tue.nl

providing details and we will investigate your claim. 


\section{EINDHOVEN UNIVERSITY OF TECHNOLOGY}

Department of Mathematics and Computer Science

\section{CASA-Report Io-66}

October 2010

\section{On modeling of curved jets of viscous}

fluid hitting a moving surface

by

A. Hlod

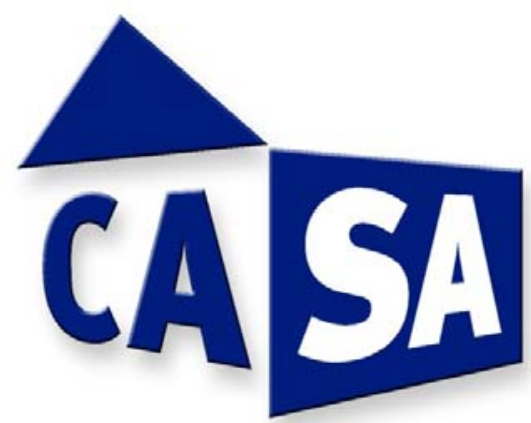

Centre for Analysis, Scientific computing and Applications

Department of Mathematics and Computer Science

Eindhoven University of Technology

P.O. Box 513

5600 MB Eindhoven, The Netherlands

ISSN: 0926-4507 



\title{
On Modeling of Curved Jets of Viscous Fluid Hitting a Moving Surface
}

A. Hlod

\begin{abstract}
A jet of Newtonian fluid can fall from the oriented nozzle onto the moving surface in three regimes. A flow regime depends on the process parameters and is characterized by the dominant effect in the momentum transfer through the jet crosssection. To model the three jet flow regimes we describe the jet by the effects of inertia, longitudinal viscosity, and gravity. The key issue is to prescribe the boundary conditions for the jet orientation, which follow from the conservation of momentum for the dynamic jet. If the jet is under tension, the principal part of the conservation of momentum equation is of hyperbolic type, and the boundary conditions for the jet shape follow from the directions of characteristics. From this we find that the boundary conditions for the jet orientation are determined by the dominant effect in the momentum transfer through the jet cross-section, which can be due to inertia, or due to viscosity. This choice of boundary conditions allows us to find the solution to the steady jet model for all parameters, and partition the parameter space between the three jet flow regimes.
\end{abstract}

\section{Introduction}

Growing interest in modeling of industrial processes (such as production of glass wool [19], high-temperature thermal isolation [1], and rotor spinning process [6], [14], [12]) requires development and study of models of a fluid jet hitting a moving surface under influence of external forces. These models are used to predict the jet shape, study jet stability, and describe the influence of the process parameters.

A configuration, in which the fluid jet hits a moving surface, is the jet of viscous fluid falling onto a moving belt under gravity. In this process the three flow regimes of the jet fall are distinguished and characterized by the convexity or concaveness

A. Hlod

Dept. of Mathematics and Computer Science, Eindhoven University of Technology, PO Box 513 5600 MB Eindhoven The Netherlands, e-mail: avhlod@gmail.com 
of the jet shape i.e. concave, vertical and convex; see Figure 1. For characterization, we consider only the major part of the jet, neglecting possible bending or unstable regions near the nozzle and the belt. A flow regime is determined by the process parameters i.e. fluid kinematic viscosity $v$, flow velocity at the nozzle $v_{\text {nozzle }}$, belt velocity $v_{\text {belt }}$ and falling height $L$.

Next, we describe each flow regime, and provide the naming based on the dominant effect in the momentum transfer through the jet cross-section; see Section 3 for details. The inertial jet has a concave shape compared to a ballistic trajectory, and occurs, among other possibilities. for small $v$ and large $v_{\text {nozzle }}$; see Figure 1(a). The viscous-inertial jet has a straight vertical shape, and among other occurs for large $v$ and $L$, and small $v_{\text {nozzle }}$; see Figure 1(b). The viscous jet has a convex shape, and occurs for large $v$ and $v_{\text {belt }}$, and small $v_{\text {nozzle }}$ and $L$; see Figure 1(c).

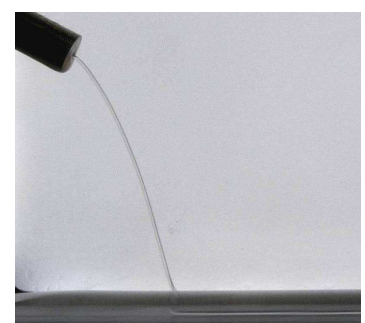

(a) Inertial (concave) jet.

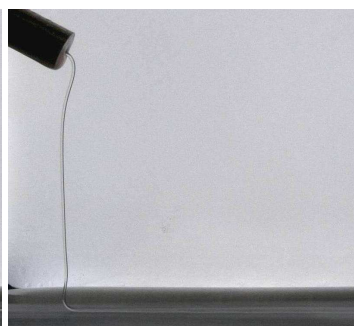

(b) Viscous-inertial (vertical) jet.

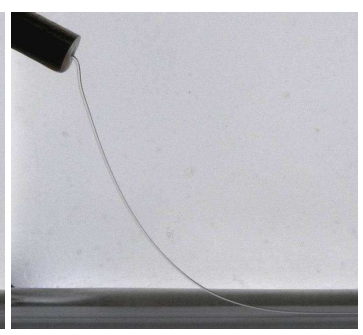

(c) Viscous (convex) jet.

Fig. 1 The three flow regimes of the jet falling onto the moving belt

In this paper we address a problem of modeling the three jet flow regimes describe above. In particular we would like to model the three regimes using as simple model as possible. To do that we use a model of [12], which includes all the essential effects (inertia, viscosity and gravity) to qualitatively describe the jet fall. Such kind of jet model is often called the string jet model analogously to the elastic strings. The system is defined by the three dimensionless quantities and the nozzle orientation.

Similar problems of the curved jets under the influence of gravity, or centrifugal and Coriolis forces but no moving surface, are described in [5, 16, 18-20]. Various aspects of the problem addressed here have been studied in $[2,12,13,17,21]$.

In this paper we start with description of the jet model, which is partly solved and transformed into a first order ODE on an unknown domain with additional scalar unknown; see Section 2. Next, we extensively discuss and motivate our choice of boundary conditions for the jet shape by studying the conservation of momentum equation for the dynamic jet; see Section 3. In Section 4 we present the partitioning of the parameter space between the three flow regimes, and perform some simulations. In Section 5 we give some conclusions. 


\section{Model}

The jet is modeled as a curve in $2 D$; see Figure 2, which is parameterized by its arc length $s$ with the origin at the nozzle $s=0$ and $s=s_{\text {end }}$ at the belt. Here $s_{\text {end }}$ is the unknown jet length. The local coordinate system with the basis of the tangent and the normal vectors $\mathbf{e}_{t}, \mathbf{e}_{n}$ is constructed at each point at the jet. The angle between the tangent vector and the horizontal direction is $\Theta$. The nozzle orientation is given by the angle $\alpha_{\text {nozzle }}$. The flow velocity in the jet is $v$. The jet at the touchdown point has the same velocity as the belt $v\left(s_{\text {end }}\right)=v_{\text {belt }}$, and the flow velocity at the nozzle is $v(0)=v_{\text {nozzle }}$. The cross-sectional area of the jet is $\mathscr{A}$.

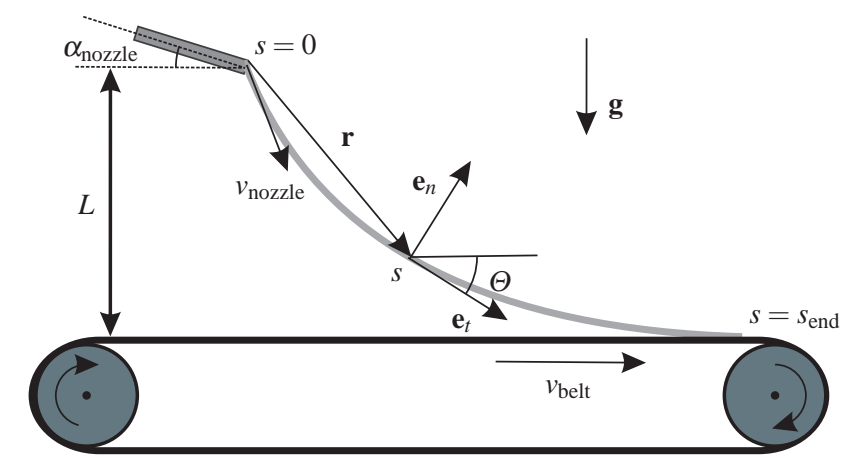

Fig. 2 The fall of the viscous jet onto the moving belt.

The system of equations describing a thin dynamical jet in $2 \mathrm{D}$ can be found $[8,22,24]$. It consists of the laws of conservations of momentum and mass, and for the stationary jet is

$$
\begin{array}{r}
\rho \mathscr{A}\left(\mathbf{r}_{s} v v_{s}+v^{2} \mathbf{r}_{s s}\right)=3 v \rho\left(v_{s} \mathscr{A} \mathbf{r}_{s}\right)_{s}+\rho \mathscr{A} \mathbf{g}, \\
(\mathscr{A} v)_{s}=0, \\
\left|\mathbf{r}_{s}\right|=1 .
\end{array}
$$

Here, $\rho$ is the fluid density and $\mathbf{g}$ is gravity. Next, we perform following manipulations

1. find $\mathscr{A}$ from (2) and substitute into (1);

2. introduce a new variable

$$
\xi=v-3 v \frac{v_{s}}{v}
$$

which stands for the momentum transfer through the jet cross-section;

3. write the components of $\mathbf{r}_{s}$ in terms of the angle $\Theta$ in the local coordinate basis $\mathbf{e}_{t}, \mathbf{e}_{n}$ (such that (3) is automatically satisfied);

4. scale the system as follow: the length is scaled with respect to $3 v / v_{\text {nozzle }}$ and the velocity with respect to $v_{\text {nozzle }}$; 
5. replace the material coordinate $s$ by the lagrangian $\tau$, according to

$$
d s=v(\tau) d \tau
$$

Then, (1) together with the boundary conditions become

$$
\begin{aligned}
& \xi_{\tau}=A \sin (\Theta), \\
& \Theta_{\tau}=\frac{A \cos (\Theta)}{\xi}, \\
& \xi=v-\frac{v_{\tau}}{v^{2}}, \\
& v(0)=1, \\
& v\left(\tau_{\text {end }}\right)=\operatorname{Dr}, \\
& \int_{0}^{\tau_{\text {end }}} \sin (\Theta(\tau)) v(\tau) d \tau=\operatorname{Re} .
\end{aligned}
$$

Here $A=3 g v / v_{\text {nozzle }}^{3}$, the Reynolds number $\operatorname{Re}=v_{\text {nozzle }} L /(3 v)$, the draw ratio $\operatorname{Dr}=$ $v_{\text {belt }} / v_{\text {nozzle }}$, and $\tau_{\text {end }}$ is the results of coordinate transformation (5) of $s_{\text {end }}$. Thus, the system is described by the three dimensionless parameters and the parameter space $\mathscr{P}$ is

$$
\mathscr{P}:=\{(A, \operatorname{Re}, \operatorname{Dr}): A>0, \operatorname{Re}>0, \operatorname{Dr}>0\} .
$$

We solve (6)-(7), using following boundary conditions for $\Theta$ that are derived in Section 3

$$
\begin{gathered}
\Theta(0)=\alpha_{\text {nozzle }} \\
\Theta\left(\tau_{\text {end }}\right)=0
\end{gathered}
$$

for the inertial and viscous jets respectively. For the viscous-inertial jet

$$
\Theta \equiv \pi / 2 \text {. }
$$

Next, we partly solve (6)-(6), (13) - (15), and find $\Theta$ and $\xi$ explicitly. After substituting the solutions for $\Theta$ and $\xi$ into (8)-(11) we arrive at the system for $v$ and $\tau_{\text {end }}$

$$
\begin{aligned}
& v-\frac{v_{\tau}}{v^{2}}= \begin{cases}w \sqrt{A^{2} \tau\left(\tau-2 \tau_{\text {end }}\right) / w^{2}+1} & \text { if convex jet, } \\
w+A \tau & \text { if vertical jet, } \\
\sqrt{A^{2} \tau^{2}+w^{2}+2 A \tau w \sin \left(\alpha_{\text {nozzle }}\right)} & \text { if concave jet, }\end{cases} \\
& v(0)=1, \\
& v\left(\tau_{\text {end }}\right)=\operatorname{Dr} \text {, } \\
& \operatorname{Re}=\left\{\begin{array}{lr}
\int_{0}^{\tau_{\text {end }}} \frac{A\left(\tau_{\text {end }}-\tau\right)}{\sqrt{A^{2} \tau\left(\tau-2 \tau_{\text {end }}\right)+w^{2}}} v(\tau) d \tau & \text { if convex jet, } \\
\int_{0}^{\tau_{\text {end }}} v(\tau) d \tau & \text { if vertical jet } \\
\int_{0}^{\tau_{\text {end }} \frac{A \tau+w \sin \left(\alpha_{\text {nozzle }}\right)}{\sqrt{A^{2} \tau^{2}+w^{2}+2 A \tau w \sin \left(\alpha_{\text {nozzle }}\right)}} v(\tau) d \tau \text { if concave jet }}
\end{array}\right.
\end{aligned}
$$


where $w=\xi(0)$ is unknown as well. The system (16) - (19) is solved using shooting method; see [12] for more details.

\section{Boundary Conditions for the Jet Shape}

In this section we extensively discuss the boundary conditions for the jet shape.

As it follows from [11], demanding the alignment of the jet at the nozzle with the nozzle orientation leads to non-existence of the solution for certain model parameters; see Figures 1(a) and 1(b) for illustration. Figures 1(b) and 1(c) suggest that tangency at the surface should be prescribed as a boundary condition in the second case, but not in the first. In such a way depending on the situation one might need to demand tangency with the belt for the viscous jet, and alignment with the nozzle orientation for the inertial jet. In this section we derive a criterion how to prescribe boundary conditions for $\mathbf{r}$. The approach presented here is applicable to the string jet model in different configurations; e.g. rotary spinning.

To determine the boundary conditions for $\mathbf{r}$, we write the dynamic conservation of momentum equation as a semi-linear partial differential equation for $\mathbf{r}$ of the form

$$
\mathbf{r}_{t t}+2 v \mathbf{r}_{s t}+v \xi \mathbf{r}_{s s}=\mathbf{r}_{t t}+2 v \mathbf{r}_{s t}+v \xi \mathbf{r}_{s s}=\tilde{\mathbf{f}},
$$

where $\tilde{\mathbf{f}}=\left(3 v\left(\mathscr{A} v_{s}\right)_{s} / \mathscr{A}-v_{t}-v v_{s}\right) \mathbf{r}_{s}+\mathbf{g}$. According to the classification [3, p. 422-423] the equation (20) is hyperbolic when $v_{s}>0$, parabolic when $v_{s}=0$, and elliptic when $v_{s}<0$.

The sign of the variable $\xi$ plays a crucial role in this equation. The quantity

$$
\rho \mathscr{A} v \xi=\rho \mathscr{A} v^{2}-3 v \rho \mathscr{A} v_{s}
$$

represents the net momentum flux (i.e. the momentum transfer per unit of time) through a cross-section due to inertia $\rho \mathscr{A} v^{2}$ and viscosity $3 v \rho \mathscr{A} v_{s}$. For a positive sign of $\xi$, the momentum flux due to inertia is larger than that due to viscosity, and for a negative sign it is the other way around.

Let us consider only the case $v_{s}>0$ throughout the jet, so that (20) is hyperbolic. We comment on the case $v_{s}<0$ in Remark 2 at the end of this section.

For hyperbolic equations it is well-known that the number of boundary conditions at each boundary should be equal to the number of the characteristics directed into the domain at this point $[10, \mathrm{p} .417]$ and $[7,15]$. An easy way to understand this follows from the concept of "domain of dependence" [3, p. 438-449].

The characteristic equation [4, p. 57] for (20) is

$$
z^{2}-2 v z+v^{2}-3 v v_{s}=0
$$

where $z$ is the velocity of a characteristic curve. Equation (22) has the solutions

$$
z_{1}=v+\sqrt{3 v v_{s}}, \text { and } z_{2}=v-\sqrt{3 v v_{s}} .
$$


The directions of the characteristics of (20) depend on the sign of $\xi$ as follows:

1. If $\xi<0$ then $z_{1}>0$ and $z_{2}<0$, i.e. one characteristic points to the left and one to the right.

2. If $\xi=0$ then $z_{1}>0$ and $z_{2}=0$, i.e. one characteristic points to the right and one is stationary.

3. If $\xi>0$ then $z_{1}>0$ and $z_{2}>0$, i.e. both characteristics point to the right.

In this problem the characteristic $z_{1}$ is identified with the information about the jet position and the characteristic $z_{2}$ is identified with the information about the jet orientation.

Next, we will state the monotonic properties of $\xi(s)$ for the steady jet. We will use these properties to determine the characteristic directions of the dynamic jet equations for $\mathbf{r}$ (20) at both jet ends. From this the boundary conditions for $\mathbf{r}$ directly follow.

Now let us consider the steady jet. By taking the inner product of (1) with $\mathbf{r}_{s}$ and using (3), we obtain

$$
\xi_{s}=\left(\mathbf{g}, \mathbf{r}_{s}\right) / v
$$

In our configuration the term $\left(\mathbf{g}, \mathbf{r}_{s}\right) / v$ is always positive (follow from the explicit solution for $\Theta$ ), and thus the function $\xi(s)$ is strictly increasing. As a consequence there are three possibilities for the sign of $\xi(s)$ :

1. $\xi(s)<0$ for $s \in\left[0, s_{\text {end }}\right]$. According to (21) viscous momentum flux dominates inertial flux everywhere in the jet. Because of that we call this flow regime viscous.

2. $\xi(s)<0$ for $s \in\left[0, s^{*}\right)$ and $\xi(s)>0$ for $s \in\left(s^{*}, s_{\text {end }}\right]$, where $\xi\left(s^{*}\right)=0$ and $s^{*} \in$ $\left[0, s_{\text {end }}\right]$. According to $(21)$, viscous momentum flux dominates at the nozzle and inertial flux dominates at the surface. Because of that we call this flow regime viscous-inertial.

3. $\xi(s)>0$ for $s \in\left[0, s_{\text {end }}\right]$. According to (21), inertial momentum flux dominates viscous flux everywhere in the jet. Because of that we call this flow regime inertial.

Thus, the sign of $\xi$ provides a classification of the three flow regimes for the jet flow.

Next, we select the boundary conditions for $\mathbf{r}$ in case of the steady jet. To do this we treat the solution of the steady jet equations as the stationary solution of the dynamic jet equations. Doing this for (20), we obtain the boundary conditions for $\mathbf{r}$ from the characteristic directions of (20), which are determined by the sign of $\xi$. Next, we treat the three jet flow regimes separately:

1. In the case of the viscous jet, both at the nozzle and at the surface one characteristic $z_{2}$ points to the left and one $z_{1}$ to the right; see Figure 3(a). Therefore, we have to prescribe one boundary condition for $\mathbf{r}$ at each end. At the nozzle $(s=0)$ we prescribe the nozzle position, and at the surface we prescribe the tangency with the surface ( $s=s_{\text {end }}$ ). The latter provides the boundary condition for the jet orientation. 


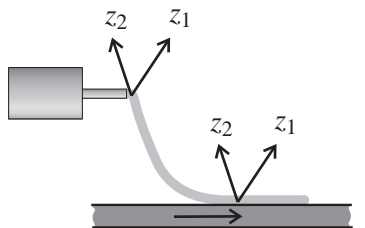

(a) Viscous jet.

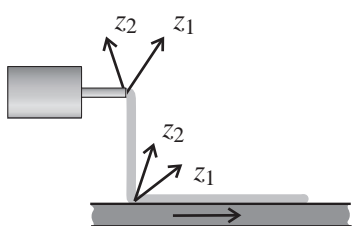

(b) Viscous-inertial jet.

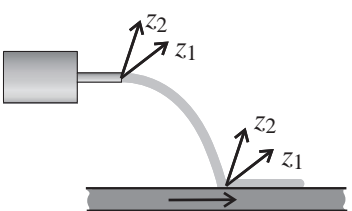

(c) Inertial jet.

Fig. 3 Characteristics directions for the three flow regimes in drag spinning.

2. In the case of the viscous-inertial jet, at the nozzle one characteristic $z_{2}$ points to the left and one $z_{1}$ to the right, and two characteristics $z_{1}$ and $z_{2}$ point to the right at the surface; see Figure 3(b). Therefore, we can only prescribe one boundary condition at the nozzle $(s=0)$, namely the nozzle position. The missing condition will be formulated in (25) further on.

3. In the case of the inertial jet, two characteristics $z_{1}$ and $z_{2}$ point to the right, both at the nozzle and at the surface; see Figure 3(c). Therefore, we prescribe two boundary conditions at the nozzle, i.e the nozzle position and orientation. The latter condition is new and provides the boundary condition for the jet orientation.

Hence, for the steady jet we appoint the nozzle position as a boundary condition for all the three flow regimes, the tangency with the surface for the viscous flow, and the nozzle orientation for the inertial flow.

Remark 1. The method of prescribing the boundary conditions for $\mathbf{r}$ according to the direction of characteristic determined by the sign of $\xi$ described above does not cover the situation if the jet or its part is under compression. For the jet under compression the equation for $\mathbf{r}$ is elliptic and the method described above is not applicable. We extend the mechanism of prescribing boundary conditions for the steady jet fully or partly under compression and prescribe the boundary conditions for $\mathbf{r}$ according to the sign of $\xi$ in the same way as described above.

Note that for the viscous-inertial jet, we prescribe only one boundary condition for the second-order differential equation (1) for $\mathbf{r}$. An extra condition follows from $\xi\left(s^{*}\right)=0$, expressing that at $s=s^{*}$ the jet should be aligned with the direction of the external force at this point, or, as follows from (1),

$$
\mathbf{r}_{s}=\frac{1}{v \xi_{s}} \mathbf{g} \text { at } \quad s=s^{*}
$$

The analysis of characteristics, as directions of information propagation, explains why the nozzle orientation influences the jet shape only in the inertial flow, and why the surface orientation influences the jet shape only in the viscous flow. In this respect, we see that:

- In viscous flow, one characteristic points into the domain at the nozzle and one at the surface. Hence, information about the direction of the surface orientation 


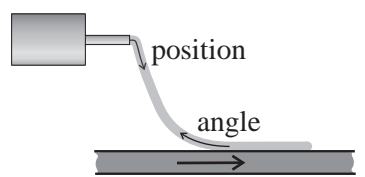

(a) Viscous jet.

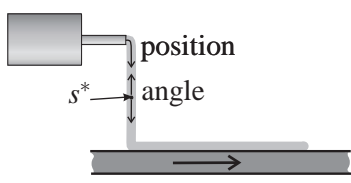

(b) Viscous-inertial jet.

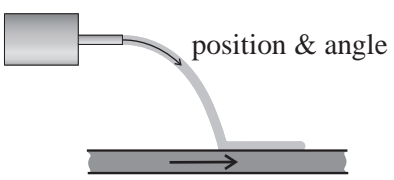

(c) Inertial jet.

Fig. 4 Directions of information propagations for the three flow regimes.

influences the jet shape; see Figure 4(a). Therefore, the surface orientation becomes relevant in viscous flow, whereas the nozzle orientation is irrelevant for the viscous jet.

- In viscous-inertial flow, only one characteristic (at the nozzle) points into the domain. Therefore, no information about the nozzle orientation or the flow orientation at the surface influences the jet shape; see Figure 4(b). Thus, in viscousinertial flow the nozzle and the surface orientations are irrelevant for the jet. The information about the orientation travels from the point $s^{*}$ towards the nozzle and the surface.

- In inertial flow, the information about the jet shape travels from the nozzle to the surface. Therefore, not only the nozzle position but also the nozzle orientation is relevant for the jet; see Figure 4(c). In addition, no information on the flow orientation travels back from the surface.

Remark 2. The dynamic equation for $\mathbf{r}$, (20), becomes elliptic when $v_{s}<0$, and in reality a steady jet might not exist [23]. In this situation the conservation of momentum (20) becomes elliptic for $\mathbf{r}$. In case $v_{s}<0$, everywhere in the jet, one has to solve a Cauchy problem for the elliptic equation. Such kind of problems are expected to be ill-posed. Analogy can be made with Hadamard's example [9, p. 234]. This example shows that a solution to a Cauchy problem for the Laplace equation does not continuously depends on the initial data in any Sobolev norm. It is possible to show that for some arbitrarily small initial data, the solution can be arbitrary large. Because of this the dynamic string model does not adequately describe the jet because it is unstable.

\section{Results}

In this section we present partitioning of the parameter space, and the jet shape evolution if one physical parameter changes.

The regions of inertial $\mathscr{P}_{\text {inert }}$, viscous-inertial $\mathscr{P}_{\mathrm{v}-\mathrm{i}}$, and viscous $\mathscr{P}_{\text {visc }}$ jets, and the borders between them, are illustrated in Figure 5. Note, that the regions of the model parameters: $\mathscr{P}_{\text {inert }}, \mathscr{P}_{\mathrm{v} \text {-i }}$ and $\mathscr{P}_{\text {visc }}$ do not intersect and cover the admissible parameter space $\mathscr{P}$.

Next, we study the evolution of the jet if one of the dimensional parameters varies as to change the flow type from viscous to viscous-inertial. For a reference 


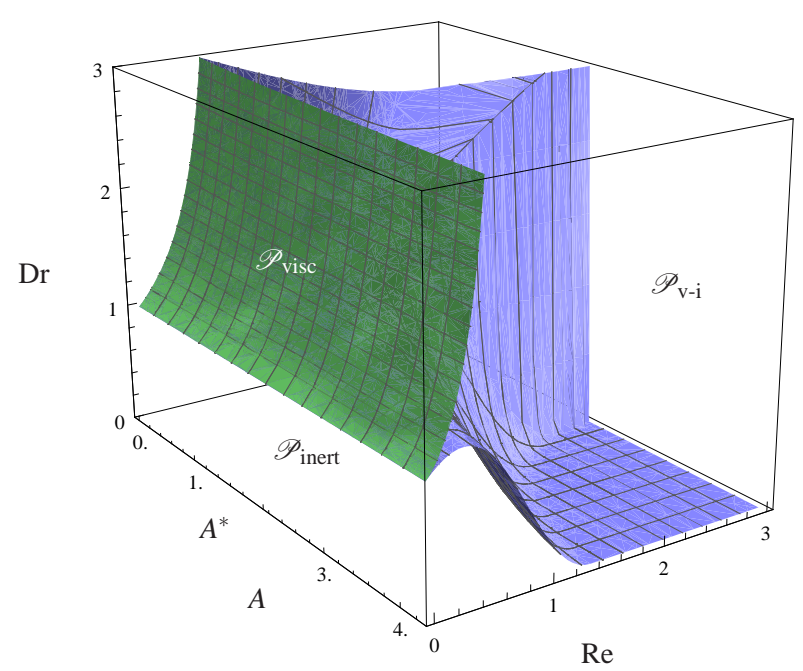

Fig. 5 Parameter regions for three flow regimes $\mathscr{P}_{\text {inert }}, \mathscr{P}_{\mathrm{v}-\mathrm{i}}$ and $\mathscr{P}_{\text {visc }}$.

configuration we take the dimensional parameters $L=1 \mathrm{~cm}, v=0.047 \mathrm{~m}^{2} / \mathrm{s}, v_{\text {belt }}=$ $1.4 \mathrm{~m} / \mathrm{s}$, and $v_{\text {nozzle }}=1 \mathrm{~m} / \mathrm{s}$, for which the jet is viscous. If we increase $L$, decrease $v$, decrease $v_{\text {belt }}$, or increase $v_{\text {nozzle }}$, eventually the jet flow changes from viscous to viscous-inertial.

Changes of the jet shape while only one of the dimensional parameters $L, v$, $v_{\text {belt }}$, or $v_{\text {nozzle }}$ vary as described above are shown in Figures 6(a), 6(b), 6(c), and 6(d), respectively. If the point $(A, \operatorname{Re}, \mathrm{Dr})$ approaches the boundary of $\mathscr{P}_{\text {visc }}$, the jet shape becomes vertical. If $(A, \operatorname{Re}, \mathrm{Dr})$ is very close to the boundary of $\mathscr{P}_{\text {visc }}$ the jet shape is almost vertical, except for the small region near the belt where the jet rapidly bends to the horizontal belt direction.

To illustrate the change of flow from inertial to viscous-inertial, while only one of the parameters $L, v, v_{\text {belt }}$, and $v_{\text {nozzle }}$ varies, we take the reference values $L=30 \mathrm{~cm}$, $v=0.2 \mathrm{~m}^{2} / \mathrm{s}, v_{\text {belt }}=2 \mathrm{~m} / \mathrm{s}$, and $v_{\text {nozzle }}=1.5 \mathrm{~m} / \mathrm{s}$. If we decrease $L$, increase $v$, increase $v_{\text {belt }}$, or decrease $v_{\text {nozzle }}$ eventually the jet flow changes from inertial to viscous-inertial.

Changes of the jet shape for $\alpha_{\text {nozzle }}=\pi / 4$, while only one of the dimensional parameters $L, v, v_{\text {belt }}$, or $v_{\text {nozzle }}$ varies as described above are shown in Figures 7(a), $7(\mathrm{~b}), 7(\mathrm{c})$, and $7(\mathrm{~d})$, respectively. If the point $(A, \mathrm{Re}, \mathrm{Dr})$ approaches the boundary of $\mathscr{P}_{\text {inert }}$, the jet shape becomes more vertical. If $(A, \operatorname{Re}, \mathrm{Dr})$ is very close to the boundary of $\mathscr{P}_{\text {inert }}$ the jet shape is almost vertical except for the small region near the belt where the jet rapidly bends from the nozzle direction to an almost vertical one. 


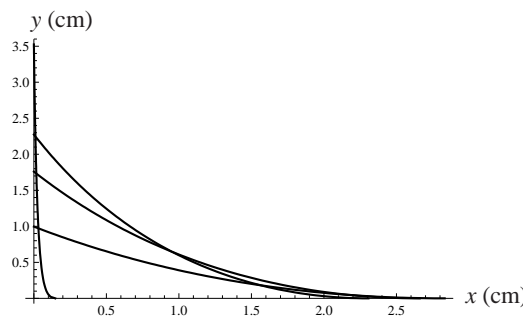

(a) Viscous jet shapes for different $L$ : $1,1.7,2.2$, and $3.5 \mathrm{~cm}$. The shape approaches the vertical as $L$ increases.

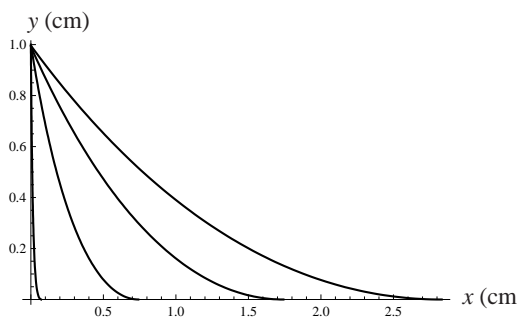

(c) Viscous jet shapes for different $v_{\text {belt }}: 1.4,1.21,1.11$, and $1.08 \mathrm{~m} / \mathrm{s}$. The shape approaches the vertical as $v_{\text {belt }}$ decreases.

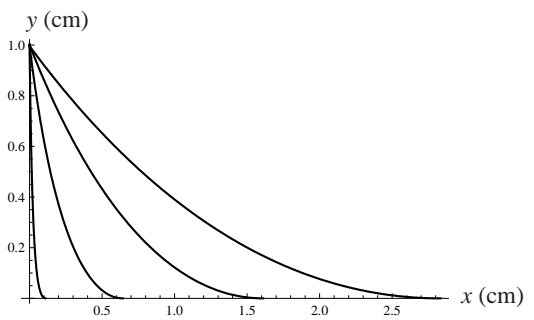

(b) Viscous jet shapes for different $v$ : $0.047,0.026,0.015$, and $0.012 \mathrm{~m}^{2} / \mathrm{s}$. The shape approaches the vertical as $v$ decreases.

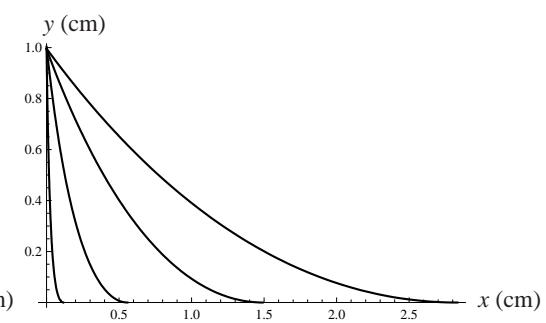

(d) Viscous jet shapes for different $v_{\text {nozzle }}: 1,1.16,1.24$, and $1.26 \mathrm{~m} / \mathrm{s}$. The shape approaches the vertical as $v_{\text {nozzle }}$ increases.

Fig. 6 Shapes of the viscous jet for different values of $L, v, v_{\text {belt }}$ and $v_{\text {nozzle. The reference values }}$ are $L=1 \mathrm{~cm}, v=0.047 \mathrm{~m}^{2} / \mathrm{s}, v_{\text {belt }}=1.4 \mathrm{~m} / \mathrm{s}$, and $v_{\text {nozzle }}=1 \mathrm{~m} / \mathrm{s}$.

\section{Conclusions}

In this paper we present a model describing the three flow regimes of the jet of viscous fluid falling onto the moving surface. The model includes effects of inertia, longitudinal viscosity, and gravity, and describes the jet for all admissible parameters. The key issue is the boundary condition for the jet orientation, which follow from the conservation of momentum for the dynamic jet. This equation is of hyperbolic type if the jet is under tension. Thus, the number of characteristics pointing inside the domain at each jet end is equal to the number of boundary conditions for $\mathbf{r}$ at this jet end. From this follows that the choice of the boundary conditions depends on the dominant effect in the momentum transfer, which can be due to inertia, or viscosity.

The way to prescribe boundary conditions for the jet orientation based on the dominant effect in the momentum transfer (described in this paper) should be used for the string type jet models when the jet is in different configuration. 


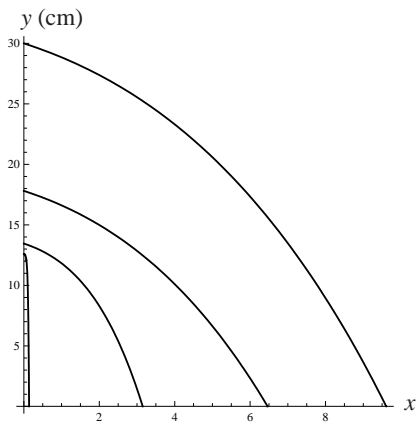

(a) Inertial jet shapes for different $L: 30,18,13$, and $12 \mathrm{~cm}$. The shape approaches the vertical as $L$ decreases.

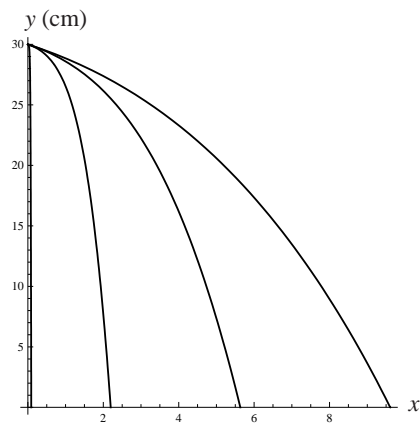

(c) Inertial jet shapes for different $v_{\text {belt }}: 2,2.57,2.86$, and $2.95 \mathrm{~m} / \mathrm{s}$. The shape approaches the vertical as $v_{\text {belt }}$ increases

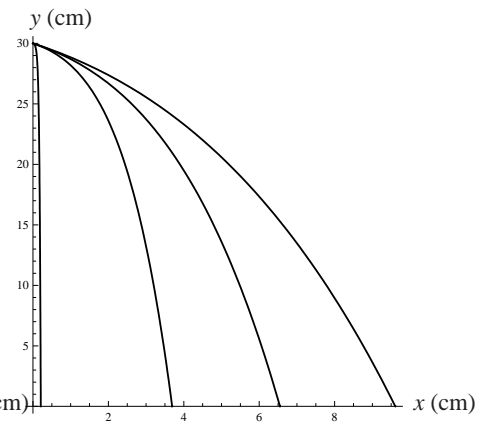

(b) Inertial jet shapes for different $v$ : $0.2,0.26,0.3$, and $0.32 \mathrm{~m}^{2} / \mathrm{s}$. The shape approaches the vertical as $v$ increases.

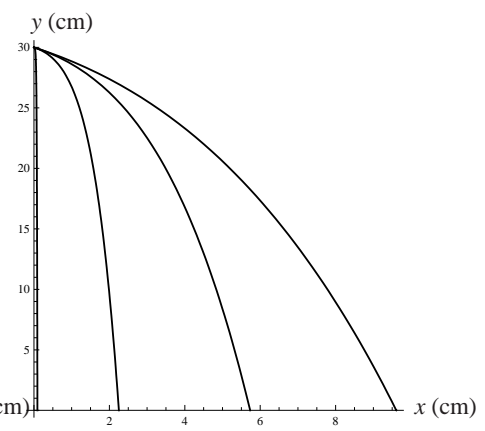

(d) Inertial jet shapes for different $v_{\text {nozzle }}: 1.5,1.4,1.36$, and 1.34 $\mathrm{m} / \mathrm{s}$. The shape approaches the vertical as $v_{\text {nozzle }}$ decreases.

Fig. 7 Shapes of the inertial jet for different values of $L, v, v_{\text {belt }}, v_{\text {nozzle }}$. The reference values are $L=30 \mathrm{~cm}, v=0.2 \mathrm{~m}^{2} / \mathrm{s}, v_{\text {belt }}=2 \mathrm{~m} / \mathrm{s}$, and $v_{\text {nozzle }}=1.5 \mathrm{~m} / \mathrm{s}$. The nozzle orientation is $\alpha_{\text {nozzle }}=$ $\pi / 4$.

Acknowledgements The author would like to acknowledge Teijin Aramid, a part of the Teijin group of companies, for providing the experimental equipment and valuable suggestions for experiments.

\section{References}

1. Breward, C., Dyson, R., Edwards, C., Metcalfe, P., Please, C., Zyskin, M.: Modelling of melt on spinning wheels. Study group report, European Study Group with Industry 49th ESGI (Oxford 29/3/2004 - 4/4/2004) (2005). Thermal Ceramics UK

2. Chiu-Webster, S., Lister, J.R.: The fall of a viscous thread onto a moving surface: a 'fluidmechanical sewing machine'. J. Fluid Mech. 569, 89-111 (2006) 
3. Courant, R., Hilbert, D.: Methods of mathematical physics. Vol. II. Wiley Classics Library. John Wiley \& Sons Inc., New York (1989). Partial differential equations, Reprint of the 1962 original, A Wiley-Interscience Publication

4. Davis, J.L.: Mathematics of wave propagation. Princeton University Press, Princeton, NJ (2000)

5. Decent, S.P., King, A.C., Wallwork, I.M.: Free jets spun from a prilling tower. Journal of Engineering Mathematics 42(3), 265 - 282 (2002)

6. den Decker, P., Knoester, H., Meerman, H., Dekker K. van Horssen, W., Vuik, C., Wesswling, P., Prokert, G., van 't Hof, B., van Beckum, F.: The rotor spinning process for fibr production. In: Proceedings of the 48th European Study Group Mathematics with Industry (Delft, 15-19 March 2004), pp. 35-48

7. Dubois, F., LeFloch, P.: Boundary conditions for nonlinear hyperbolic systems of conservation laws. J. Differential Equations 71(1), 93-122 (1988)

8. Entonov, V.M., Yarin, A.L.: Dynamical equation for a liquid jet. Translated from Izvestiya Akademii Nauk SSSR, Mekhanika Zhidkosti i Gaza (5), 11-18 (1980)

9. Evans, L.C.: Partial differential equations, Graduate Studies in Mathematics, vol. 19. American Mathematical Society, Providence, RI (1998)

10. Godlewski, E., Raviart, P.A.: Numerical approximation of hyperbolic systems of conservation laws, Applied Mathematical Sciences, vol. 118. Springer-Verlag, New York (1996)

11. Götz, T., Klar, A., Unterreiter, A., Wegener, R.: Numerical evidence for the non-existence of stationary solutions of the equations describing rotational fiber spinning. Mathematical Models and Methods in Applied Sciences 18(10), 1829 - 1844 (2008)

12. Hlod, A.: Curved jets of viscous fluid: Interactions with a moving wall. Ph.D. thesis, Eindhoven University of Technology (2009)

13. Hlod, A., Aarts, A., v.d. Ven, A., Peletier, M.: Mathematical model of falling of a viscous jet onto a moving surface. European Journal of Applied Mathematics 18, 659-677 (2007)

14. Kolk, E.: Modelling of melt on spinning wheels. Interim report, TU Delft (2005)

15. Kreiss, H.O.: Initial boundary value problems for hyperbolic systems. Comm. Pure Appl. Math. 23, 277-298 (1970)

16. Marheineke, N., Wegener, R.: Asymptotic model for the dynamics of curved viscous fibres with surface tension. Journal of Fluid Mechanics 622(-1), 345-369 (2009)

17. Morris, S.W., Dawes, J.H.P., Ribe, N.M., Lister, J.R.: Meandering instability of a viscous thread. Physical Review E (Statistical, Nonlinear, and Soft Matter Physics) 77(6), 066218 (2008)

18. Panda, S.: The dynamics of viscous fibers. Ph.D. thesis, Technische Universität Kaiserslautern (2006)

19. Panda, S., Marheineke, N., Wegener, R.: Systematic derivation of an asymptotic model for the dynamics of curved viscous fibers. Mathematical Methods in the Applied Sciences 31(10), $1153-1173$ (2008)

20. Parau, E.I., Decent, S., Simmons, M., Wong, D.C.Y., King, A.C.: Nonlinear viscous liquid jets from a rotating orifice. Journal of Engineering Mathematics 57(2), 159-179 (2007)

21. Ribe, N.M., Lister, J.R., Chiu-Webster, S.: Stability of a dragged viscous thread: Onset of stitching in a fluid-mechanical sewing machine. Phys. Fluids 18, 124,105-1-8 (2006)

22. Roos, J.P., Schweigman, C., Timman, R.: Mathematical formulation of the laws of conservation of mass and energy and the equation of motion for a moving thread. Journal of Engineerimg Mathematics 7(2), 139-146 (1973)

23. Taylor, G.: Instability of jets, threads, and sheets of viscous fluid. In: Proceedings of the 12th International Congress of Applied mechnaics (Stanford, 1968), pp. 382-388. Springer-Verlag (1969)

24. Yarin, A.L.: Free liquid jets and films: hydrodynamics and rheology. Interaction of Mechanics and Mathematics Series. Longman Scientific \& Technical, Harlow (1993) 
PREVIOUS PUBLICATIONS IN THIS SERIES:

\begin{tabular}{|c|c|c|c|}
\hline Number & Author(s) & Title & Month \\
\hline $10-62$ & $\begin{array}{l}\text { D.J. Bekers } \\
\text { S.J.L. van Eijndhoven }\end{array}$ & $\begin{array}{l}\text { Spectral analysis of } \\
\text { integral-differential } \\
\text { operators applied in linear } \\
\text { antenna modeling }\end{array}$ & Oct. 'IO \\
\hline $10-63$ & L.M.J. Florack & $\begin{array}{l}\text { Scale space } \\
\text { representations locally } \\
\text { adapted to the geometry of } \\
\text { base and target manifold }\end{array}$ & Oct. 'Io \\
\hline $10-64$ & $\begin{array}{l}\text { F. Schilder } \\
\text { R. Christensen } \\
\text { J. Sylvest } \\
\text { K. Kumar } \\
\text { M. Rudnaya } \\
\text { V. Savcenco } \\
\text { B. Sobiegraj } \\
\text { C. Please } \\
\text { R. Style }\end{array}$ & $\begin{array}{l}\text { Design parameters for a } \\
\text { siphon system }\end{array}$ & Oct. 'Io \\
\hline $10-65$ & $\begin{array}{l}\text { A. Hlod } \\
\text { A.A.F. van de Ven } \\
\text { M.A. Peletier }\end{array}$ & $\begin{array}{l}\text { A model of rotary spinning } \\
\text { process }\end{array}$ & Oct. 'Io \\
\hline I0-66 & A. Hlod & $\begin{array}{l}\text { On modeling of curved jets } \\
\text { of viscous fluid hitting a } \\
\text { moving surface }\end{array}$ & Oct. 'Io \\
\hline
\end{tabular}

